\title{
Sensibilidade de cultivares de mandioca ao herbicida mesotrione $\mathrm{e}^{1,2}$
}

\author{
Cassava cultivars sensibility to mesotrione herbicide
}

\author{
Hellen Martins Silveira ${ }^{3}$; Daniel Valadão Silva ${ }^{4}$; José Barbosa dos Santos ${ }^{5}$; Manoel Delintro \\ Castro Neto ${ }^{4}$; Evander Alves Ferreira ${ }^{6}$; Felipe Paolinelli de Carvalho ${ }^{4}$; Antonio Alberto Silva ${ }^{7}$; \\ Tocio Sediyama $^{7}$
}

Resumo - O manejo inadequado das plantas daninhas é um dos principais fatores responsáveis pela baixa produtividade média de raízes da mandioca no Brasil. Nessa cultura, o método mecânico de controle das plantas daninhas é o mais utilizado em decorrência da baixa disponibilidade de herbicidas registrados para a mandioca. Na busca de alternativas para este problema este trabalho que teve como objetivo avaliar a tolerância de cultivares de mandioca à aplicação em pós-emergência de mesotrione. Para isso foi conduzido um experimento em casa de vegetação, em delineamento de blocos casualizados com quatro repetições. Adotou-se arranjo fatorial em esquema $5 \times 5$, constituído pela combinação de cinco doses do herbicida mesotrione e cinco cultivares de mandioca. Avaliou-se semanalmente a fitotoxicidade, altura, diâmetro de caule e número de folhas das plantas, e aos 35 dias após a aplicação (DAA) coletaram-se as plantas para determinação da matéria seca e área foliar das plantas. Os maiores valores de intoxicação visual foram observados aos 14 e 28 DAA, provocados pelas maiores doses do mesotrione. Estes sintomas consistiram em leve branqueamento das folhas mais novas das plantas. Aos 35 DAA as plantas apresentaram recuperação dos sintomas, principalmente pelo surgimento de novas folhas. Em nenhuma das doses avaliadas o mesotrione afetou as variáveis: altura, diâmetro do caule, número de folhas, área foliar e acúmulo de matéria seca de folha, caule, raízes e total; evidenciando a tolerância dos cultivares de mandioca ao herbicida.

Palavras-chave: Manihot esculenta, crescimento, seletividade, manejo químico

Abstract - Unsuitable weed management is one of the main factors responsible for low mean yield of cassava (Manihot esculenta Crantz) in Brazil. In this crop, mechanical weed control is the most commonly used, due to the lack of registered herbicides for cassava crop. In the search for alternatives to this problem, this work aimed to evaluate the tolerance level of cassavas cultivars to mesotrione herbicide applied in post emergence. A experiment was conducted, in green house conditions, in randomized blocks with four replications. A factorial scheme $5 \times 5$ was adopted, consisting of the combination of five dosages of mesotrione herbicides and five cassava

\footnotetext{
${ }^{1}$ Recebido para publicação em 23/12/2011 e aceito em 22/02/2012.

${ }^{2}$ Parte da dissertação de mestrado do segundo autor defendida pelo Programa de Pós Graduação em Produção Vegetal da Universidade Federal dos Vales do Jequitinhonha e Mucuri;

${ }^{3}$ Mestranda do Programa de Pós Graduação em Fitotecnia da Universidade Federal de Viçosa. Avenida Peter Henry Rolfs, s/n, Campus UFV, 36.570-000, Viçosa, Minas Gerais, Brasil. hellenufv@ hotmail.com;

${ }^{4}$ Doutorando do Programa de Pós Graduação em Fitotecnia da Universidade Federal de Viçosa - MG;

${ }^{5}$ Docente, Programa de Pós Graduação em Produção Vegetal, Universidade Federal dos Vales do Jequitinhonha e Mucuri - Rodovia MGT 367 - Km 583, nº 5000 - Alto da Jacuba, Diamantina-MG - Brasil;

${ }^{6}$ Pós doutorando, Programa de Pós Graduação em Produção Vegetal, Universidade Federal dos Vales do Jequitinhonha e Mucuri;

${ }^{7}$ Docente do Departamento de Fitotecnia da Universidade Federal de Viçosa.
} 


\section{Silveira et al.}

cultivars. Intoxication of cassava plants, height, stem diameter and number of leaves of plants were evaluated weekly and 35 days after application (DAA) plants were harvested for determining dry matter and leaf area.n. The highest values for visual intoxication were observed at 14 and 28 days after the herbicide application (DAA) and caused by the highest doses of mesotrione herbicide. These symptoms consisted of whiteness in new leaves. At 35 days, plants showed signals of recovery, mainly due the sprout of new leaves. The herbicide did not affected the height, stem diameter, number of leaves, foliar area and dry matter accumulation of leaves, stems, roots and in total characteristics, in any of the evaluated doses. This is an evidence of cassava cultivars tolerance to mesotrione herbicide.

Keywords: Manihot esculenta, growth, selectivity, chemical control

\section{Introdução}

A mandioca (Manihot esculenta Crantz) constitui um dos componentes básicos da dieta alimentar da população brasileira desempenhando importante papel econômico e social. É cultivada em todo território nacional, devido principalmente a sua capacidade de usar eficientemente os recursos água, luz e nutrientes e ter grande adaptação a solos de baixa fertilidade (Cardoso \& Souza, 2002). Essa eficiência pode ser comprometida quando há presença de plantas daninhas na área de cultivo. A competição pelos recursos exercida por essas plantas reduz o crescimento $\mathrm{e}$ desenvolvimento da mandioca, sendo recomendado manter a área de cultivo livre de plantas daninhas no período inicial, que varia de 60 a 120 dias após o plantio (Moura, 2000 e Biffe et al., 2010).

Dentre os métodos de controle de plantas daninhas, o controle químico se caracteriza por permitir a intervenção em grandes áreas com pouca dependência de mãode-obra e rapidez na aplicação. Para a cultura da mandioca encontram-se registrados cinco ingredientes ativos (MAPA, 2011). Dentre esses, quatro (ametryn, metribuzin, isoxaflutole e o clomazone) são pré-emergentes e somente um (clethodim) é registrado para aplicação em pós-brotação da mandioca, apresentando atividade somente sobre plantas daninhas gramíneas. Esse reduzido número de herbicidas limita muito as possibilidades de manejo pelo produtor, visto que em recentes levantamentos foram identificados dezenas de espécies de plantas daninhas, de vários gêneros e famílias, que infestam os mandiocais brasileiros (Carvalho et al., 2004; Johanns \& Contiero, 2006; Albuquerque et al.,2008; Guglieri et al., 2009; Pinotti et al., 2010; Biffe et al., 2010).

Dentre os novos herbicidas no mercado, o mesotrione destaca-se por ser de ação sistêmica, indicado para o controle em pósemergência das plantas infestantes nas culturas do milho e cana-de-açúcar. Pertence ao grupo químico das tricetonas, atua sobre as plantas daninhas inibindo a biossíntese de carotenóides, através da interferência na atividade da enzima HPPD (4-hidroxifenilpiruvato-dioxigenase) nos cloroplastos (Rodrigues \& Almeida, 2005).

Considerando que há diferenciação dos genótipos de mandioca quanto à seletividade a herbicidas, objetivou-se com este trabalho avaliar a influência do herbicida mesotrione aplicado em pós-brotação da cultura sobre o crescimento inicial de cinco cultivares de mandioca.

\section{Material e Métodos}

O experimento foi conduzido em casa de vegetação utilizando-se um Latossolo Vermelho-Amarelo Distrófico típico, textura argilosa (56\% argila, $6 \%$ silte e $38 \%$ areia). A análise química do solo apresentou $\mathrm{pH}$ (água) de 5,4; teor de matéria orgânica de 1,8 daq $\mathrm{kg}^{-}$ ${ }^{1}$; $\mathrm{P}, \mathrm{K}, \mathrm{Ca}, \mathrm{Mg}, \mathrm{Al}, \mathrm{H}+\mathrm{Al}$ e $\mathrm{CTC}_{\text {efetiva }}$ de 1,4 ; $10 ; 0,5 ; 0,2 ; 0,4 ; 4,4$ e 1,7 cmolc $\mathrm{dm}^{-3}$ 
Silveira et al.

respectivamente. Para adequação do substrato quanto à nutrição, foram aplicados o equivalente a $300 \quad \mathrm{~kg}^{\mathrm{ha}}{ }^{-1}$ de calcário dolomítico, $220 \mathrm{~kg} \mathrm{ha}^{-1}$ de super fosfato simples e $40 \mathrm{~kg} \mathrm{ha}^{-1}$ de cloreto de potássio. A adubação nitrogenada foi realizada em cobertura aos 30 dias após a emergência da cultura, com $40 \mathrm{~kg} \mathrm{ha}^{-1}$ de uréia previamente dissolvida em água. As irrigações foram realizadas diariamente, por sistema automático de microaspersão.

O delineamento experimental utilizado foi $o$ de blocos casualizados com quatro repetições. Cada vaso com capacidade volumétrica de $12 \mathrm{dm}^{3}$, contendo amostra de solo, representou uma unidade experimental. Adotou-se arranjo fatorial em esquema 5x5, constituído pela combinação de 5 doses do herbicida mesotrione: $0 ; 0,072 ; 0,108 ; 0,144 \mathrm{e}$ $0,216 \mathrm{~g} \mathrm{ha}^{-1}$, equivalente a 0,$0 ; 0,5,0,75,1 \mathrm{e}$ 1,5 vezes a dose comercial recomendada (MAPA, 2011) e 5 cultivares de mandioca (Cacau UFV, Platina, Coqueiro, Coimbra e IAC-12).

Efetuaram-se o plantio das manivas com tamanho médio de $20 \mathrm{~cm}$ (5 gemas) em vaso no mês de março de 2011, com brotação visível cinco dias após o plantio. A aplicação dos herbicidas foi realizada aos 30 dias após o plantio, quando as plantas de mandioca apresentavam cerva de $30 \mathrm{~cm}$ de altura, conforme recomendado por Moura (2000) e Albuquerque et al. (2008) para o início de controle das plantas daninhas na cultura. Para isso, utilizou-se um pulverizador costal pressurizado à $\mathrm{CO}_{2}$, com pressão constante de $200 \mathrm{kPa}$, equipado com uma barra de duas pontas de indução de ar TTI 11002, trabalhando a uma altura de $50 \mathrm{~cm}$ do alvo, com velocidade de $1 \mathrm{~m}$ segundo $^{-1}$, atingindo faixa aplicada de $50 \mathrm{~cm}$ de largura, e propiciando volume de calda de $150 \mathrm{~L} \mathrm{ha}^{-1}$.

As avaliações de intoxicação visual da cultura foram realizadas aos 7, 14, 28 e 35 dias após a aplicação dos herbicidas (DAA). Para isso utilizou-se uma escala percentual de notas variando entre 0 (zero) e 100 (cem), onde 0 implicou ausência de quaisquer injúrias e 100, a morte da planta.

Aos 7, 14, 28 e 35 DAA determinou-se o número de folhas (NF), diâmetro do caule (DC) e altura das plantas (AP) e na última avaliação a área foliar (AF). Além disso, aos 35 DAA, todo o material vegetal foi colhido, separado em folha, caule e raízes sendo posteriormente seco em estufa com circulação forçada de ar a $65^{\circ} \mathrm{C}$, até atingir peso constante para determinação da massa da matéria seca.

Os dados relativos à porcentagem de intoxicação das plantas de mandioca sofreram transformação para raiz quadrada de $\mathrm{x}+1$, antes de serem submetidos à análise de variância e teste de agrupamento de SkottKnott.

Os dados das outras variáveis avaliadas foram submetidos à análise de variância, desdobrando-se todas as interações possíveis. Optou-se também por analisar cada época de avaliação separadamente. As médias, quando significativas, foram comparadas pelo teste de Tukey a 5\% de probabilidade.

\section{Resultados e Discussão}

As notas médias para intoxicação visual promovida pelas doses do herbicida aos 7, 14, 28 e 35 DAA estão apresentadas na Tabela 1. Verifica-se, que aos 7 DAA o cultivar IAC-12 apresentava a maior sensibilidade ao mesotrione, enquanto que para os demais genótipos somente as maiores doses do herbicida acarretaram em sintomas de injúrias mais evidentes, que consistiam em leve branqueamento das folhas novas das plantas. Esse resultado também foi observado para a maior dose aos 14, 28 e 35 DAA, exceto para a Platina aos 14 DAA, a qual apresentava os menores valores entre os cultivares.

Valores de intoxicação acima de $20 \%$ foram encontrados somente para os cultivares 
Silveira et al.

Cacau-UFV e IAC-12, sendo que para os demais cultivares esses valores ficaram abaixo dessa faixa. Esses valores podem ser considerados de baixa toxicidade, pois Oliveira Jr. et al. (2001) em trabalho com o herbicida clomazone, pertencente ao mesmo mecanismo de ação do mesotrione, encontraram valores de intoxicação visual superiores a $40 \%$, sem, no entanto, causar reduções significativas no estande, altura de plantas e produtividade de raízes da mandioca.

Tabela 1 - Porcentagem de intoxicação em variedades de mandioca aos 7, 14, 28 e 35 dias após a aplicação (DAA) do mesotrione.

\begin{tabular}{llrrrr}
\hline \multirow{2}{*}{ Cultivares } & Dose $\left(\mathbf{g}\right.$ i.a. ha $\left.{ }^{-1}\right)$ & \multicolumn{4}{c}{ Dias após aplicação (DAA) } \\
\cline { 3 - 6 } & & 7 DAA & 14 DAA & 28 DAA & 35 DAA \\
\hline \multirow{3}{*}{ Cacau UFV } & Mesotrione $(0,072)$ & $10,0 \mathrm{~A}$ & $7,5 \mathrm{C}$ & $7,5 \mathrm{~B}$ & $7,5 \mathrm{~B}$ \\
& Mesotrione $(0,108)$ & $6,3 \mathrm{~B}$ & $10,0 \mathrm{~B}$ & $10,0 \mathrm{~B}$ & $10,0 \mathrm{~A}$ \\
& Mesotrione $(0,144)$ & $11,3 \mathrm{~A}$ & $12,5 \mathrm{~B}$ & $16,3 \mathrm{~A}$ & $7,5 \mathrm{~B}$ \\
& Mesotrione $(0,216)$ & $13,8 \mathrm{~A}$ & $21,3 \mathrm{~A}$ & $20,0 \mathrm{~A}$ & $15,0 \mathrm{~A}$ \\
\hline \multirow{3}{*}{ Coimbra } & Mesotrione $(0,072)$ & $5,0 \mathrm{~B}$ & $3,8 \mathrm{D}$ & $3,8 \mathrm{~B}$ & $3,8 \mathrm{~B}$ \\
& Mesotrione $(0,108)$ & $3,8 \mathrm{~B}$ & $6,3 \mathrm{C}$ & $6,3 \mathrm{~B}$ & $8,8 \mathrm{~A}$ \\
& Mesotrione $(0,144)$ & $12,5 \mathrm{~A}$ & $12,5 \mathrm{~B}$ & $13,8 \mathrm{~B}$ & $13,8 \mathrm{~A}$ \\
& Mesotrione $(0,216)$ & $13,8 \mathrm{~A}$ & $18,8 \mathrm{~A}$ & $17,5 \mathrm{~A}$ & $15,0 \mathrm{~A}$ \\
\hline \multirow{3}{*}{ Coqueiro } & Mesotrione $(0,072)$ & $1,3 \mathrm{C}$ & $2,5 \mathrm{D}$ & $2,5 \mathrm{~B}$ & $6,3 \mathrm{~B}$ \\
& Mesotrione $(0,108)$ & $7,5 \mathrm{~A}$ & $10,0 \mathrm{~B}$ & $6,3 \mathrm{~B}$ & $6,3 \mathrm{~B}$ \\
& Mesotrione $(0,144)$ & $6,3 \mathrm{~B}$ & $15,0 \mathrm{~B}$ & $10,0 \mathrm{~B}$ & $8,8 \mathrm{~A}$ \\
& Mesotrione $(0,216)$ & $15,0 \mathrm{~A}$ & $17,5 \mathrm{~A}$ & $18,8 \mathrm{~A}$ & $18,8 \mathrm{~A}$ \\
\hline \multirow{2}{*}{ IAC-12 } & Mesotrione $(0,072)$ & $8,8 \mathrm{~A}$ & $11,3 \mathrm{~B}$ & $12,5 \mathrm{~B}$ & $12,5 \mathrm{~A}$ \\
& Mesotrione $(0,108)$ & $11,3 \mathrm{~A}$ & $11,3 \mathrm{~B}$ & $10,0 \mathrm{~B}$ & $5,0 \mathrm{~B}$ \\
& Mesotrione $(0,144)$ & $17,5 \mathrm{~A}$ & $23,8 \mathrm{~A}$ & $22,5 \mathrm{~A}$ & $12,5 \mathrm{~A}$ \\
& Mesotrione $(0,216)$ & $16,3 \mathrm{~A}$ & $27,5 \mathrm{~A}$ & $23,8 \mathrm{~A}$ & $13,8 \mathrm{~A}$ \\
\hline \multirow{2}{*}{ Platina } & Mesotrione $(0,072)$ & $3,8 \mathrm{~B}$ & $3,8 \mathrm{D}$ & $5,0 \mathrm{~B}$ & $6,3 \mathrm{~B}$ \\
& Mesotrione $(0,108)$ & $8,8 \mathrm{~A}$ & $6,3 \mathrm{C}$ & $5,0 \mathrm{~B}$ & $3,8 \mathrm{~B}$ \\
& Mesotrione $(0,144)$ & $11,3 \mathrm{~A}$ & $11,3 \mathrm{~B}$ & $11,3 \mathrm{~B}$ & $8,8 \mathrm{~A}$ \\
& Mesotrione $(0,216)$ & $11,3 \mathrm{~A}$ & $15,0 \mathrm{~B}$ & $16,3 \mathrm{~A}$ & $15,0 \mathrm{~A}$ \\
\hline
\end{tabular}

- Médias seguidas por mesmas letras maiúsculas na coluna não diferem entre si pelo teste de Scott-Knott $(\mathrm{p} \leq 0,05)$.

Para a altura de plantas da mandioca (AP), constatou-se interação entre os fatores cultivares e doses aos 14 e 28 DAA, enquanto que aos 7 e 35 DAA somente o fator cultivares foi significativo (Tabela 2). O cultivar Platina foi o único genótipo afetado pelo mesotrione, sendo que aos 14 e 28 DAA constatou-se para dose de $0,144 \mathrm{~g} \mathrm{ha}^{-1}$ reduções de aproximadamente $20,0 \%$ em relação à testemunha, não diferindo significativamente das demais doses. Essas diferenças não foram provavelmente verificadas na última avaliação devido à recuperação das plantas pela emissão de novas folhas.

Em avaliação de nove cultivares de mandioca feita por Vidigal Filho et al. (2000), verificou-se que a característica altura de planta está diretamente relacionada com a produção final da parte aérea da cultura. Biffe et al. (2010) e Oliveira Jr. (2001) não relataram 
Silveira et al.

reduções na AP da mandioca para a aplicação e clomazone, corroborando com os resultados em pré-emergência dos herbicidas isoxaflutole desse estudo.

Tabela 2 - Altura de plantas (cm) de cultivares de mandioca aos 7, 14, 28 e 35 dias após a aplicação (DAA) de mesotrione.

\begin{tabular}{|c|c|c|c|c|c|c|}
\hline \multirow{3}{*}{ Cultivares } & 7 DAA & \multicolumn{5}{|c|}{14 DAA } \\
\hline & \multirow{2}{*}{ Média } & \multicolumn{5}{|c|}{ Dose (g i.a. ha $\left.{ }^{-1}\right)$} \\
\hline & & $\mathbf{0}$ & 0,072 & 0,108 & 0,144 & 0,216 \\
\hline Cacau UFV & $37,2 \mathrm{~A}$ & $67,8 \mathrm{Aa}$ & $59,0 \mathrm{Aa}$ & $70,3 \mathrm{Aa}$ & $73,5 \mathrm{Aa}$ & $67,3 \mathrm{Aa}$ \\
\hline Coimbra & $31,2 \mathrm{~B}$ & $69,3 \mathrm{Aa}$ & $66,8 \mathrm{Aa}$ & $72,8 \mathrm{Aa}$ & $66,3 \mathrm{Aa}$ & $77,3 \mathrm{Aa}$ \\
\hline Coqueiro & $39,5 \mathrm{~A}$ & $83,5 \mathrm{Aa}$ & $67,5 \mathrm{Aa}$ & $66,0 \mathrm{Aa}$ & $73,3 \mathrm{Aa}$ & $66,5 \mathrm{Aa}$ \\
\hline IAC-12 & $36,5 \mathrm{AB}$ & $78,8 \mathrm{Aa}$ & $79,0 \mathrm{Aa}$ & $63,5 \mathrm{Aa}$ & $59,5 \mathrm{Aa}$ & $71,3 \mathrm{Aa}$ \\
\hline Platina & $35,4 \mathrm{AB}$ & $66,0 \mathrm{Aab}$ & $73,8 \mathrm{Aab}$ & $81,8 \mathrm{Aab}$ & $53,3 \mathrm{Ab}$ & 79,8 Aab \\
\hline CV (\%) & 18,82 & \multicolumn{5}{|c|}{------------------------ 15,15------------------------ } \\
\hline \multirow{3}{*}{ Cultivares } & \multicolumn{5}{|c|}{28 DAA } & 35 DAA \\
\hline & \multicolumn{5}{|c|}{ Dose (g i.a. ha $\left.{ }^{-1}\right)$} & \multirow{2}{*}{ Média } \\
\hline & $\mathbf{0}$ & 0,072 & 0,108 & 0,144 & 0,216 & \\
\hline Cacau UFV & $76,0 \mathrm{Aa}$ & $66,0 \mathrm{Aa}$ & $81,0 \mathrm{Aa}$ & $80,8 \mathrm{Aa}$ & $76,0 \mathrm{Aa}$ & $84,2 \mathrm{~A}$ \\
\hline Coimbra & $74,8 \mathrm{Aa}$ & $74,5 \mathrm{Aa}$ & $79,0 \mathrm{Aa}$ & $68,5 \mathrm{Aa}$ & 79,3 Аа & $79,4 \mathrm{~A}$ \\
\hline Coqueiro & $91,3 \mathrm{Aa}$ & 73,5 $\mathrm{Aa}$ & $69,8 \mathrm{Aa}$ & $79,0 \mathrm{Aa}$ & $69,5 \mathrm{Aa}$ & $86,8 \mathrm{~A}$ \\
\hline IAC-12 & $83,8 \mathrm{Aa}$ & $84,5 \mathrm{Aa}$ & $68,5 \mathrm{Aa}$ & $68,0 \mathrm{Aa}$ & $74,5 \mathrm{Aa}$ & $80,2 \mathrm{~A}$ \\
\hline Platina & 73,3 Aab & $82,8 \mathrm{Aab}$ & 89,3 Aab & $64,0 \mathrm{Ab}$ & $87,3 \mathrm{Aab}$ & $86,2 \mathrm{~A}$ \\
\hline $\mathrm{CV}(\%)$ & & 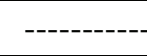 & $-14,83$ & ---- & & 13,49 \\
\hline
\end{tabular}

- Médias seguidas por mesmas letras maiúsculas na coluna e por mesmas letras minúsculas na linha não diferem entre si pelo teste de Tukey $(\mathrm{p} \leq$ 0,05). CV: Coeficiente de Variação

O diâmetro do caule (DC) apresentou cultivar Platina apresentou baixos valores aos diferenças significativas de acordo com os 14 DAA para $0,144 \mathrm{~g} \mathrm{ha}^{-1}$, apesar de não se cultivares de mandioca. De modo geral, o IAC- diferenciar significativamente das demais 12 obteve os menores valores nesta variável e o (Tabela 3 ).

Tabela 3 - Diâmetro do caule $(\mathrm{mm})$ de cultivares de mandioca aos 7, 14, 28 e 35 dias após a aplicação (DAA) de mesotrione.

\begin{tabular}{|c|c|c|c|c|c|c|c|c|}
\hline \multirow{3}{*}{ Cultivares } & 7 DAA & \multicolumn{5}{|c|}{14 DAA } & \multirow{3}{*}{$\begin{array}{l}28 \text { DAA } \\
\text { Média }\end{array}$} & \multirow{3}{*}{$\begin{array}{c}35 \text { DAA } \\
\text { Média }\end{array}$} \\
\hline & \multirow{2}{*}{ Média } & \multicolumn{5}{|c|}{ Dose (g i.a. ha-1) } & & \\
\hline & & 0 & 0,072 & 0,108 & 0,144 & 0,216 & & \\
\hline Cacau UFV & $6,7 \mathrm{AB}$ & $8,6 \mathrm{ABa}$ & $8,4 \mathrm{ABa}$ & $9,1 \mathrm{ABCa}$ & $9,0 \mathrm{ABCa}$ & $8,8 \mathrm{ABa}$ & $8,4 \quad \mathrm{~B}$ & $9,0 \mathrm{AB}$ \\
\hline Coimbra & $6,3 \quad \mathrm{~B}$ & $7,6 \quad \mathrm{Ba}$ & $7,4 \quad \mathrm{Ba}$ & $7,8 \quad \mathrm{BCa}$ & $8,0 \mathrm{ABCa}$ & $8,6 \mathrm{ABa}$ & $8,3 \quad \mathrm{~B}$ & $8,5 \quad B$ \\
\hline Coqueiro & $7,1 \mathrm{~A}$ & $10,0 \mathrm{ABa}$ & $8,8 \mathrm{ABa}$ & $8,9 \mathrm{ABCa}$ & $9,3 \mathrm{ABa}$ & $8,1 \mathrm{ABa}$ & $9,0 \mathrm{AB}$ & $9,4 \mathrm{AB}$ \\
\hline IAC-12 & $4,9 \mathrm{C}$ & $7,0 \quad \mathrm{Ba}$ & $7,0 \quad \mathrm{Ba}$ & 6,9 & 6,0 & $6,6 \quad \mathrm{Ba}$ & $6,8 \quad \mathrm{C}$ & $7,0 \quad \mathrm{C}$ \\
\hline Platina & 7,0 AB & 8,3 ABab & 9,4 ABab & 9,9 ABab & $7,3 \quad \mathrm{BCb}$ & 9,6 ABab & $9,6 \mathrm{~A}$ & $9,8 \mathrm{~A}$ \\
\hline $\mathrm{CV}(\%)$ & 14,37 & --- & - & ----12,10- & (1) & - & 15,14 & 12,64 \\
\hline
\end{tabular}


Silveira et al.

$\mathrm{Na}$ comparação entre os cultivares quanto ao número de folhas (NF) observa-se que a IAC-12 e Coimbra obtiveram os maiores valores dessa variável nas avaliações aos 7, 14 e 28 DAA (Tabela 4). No entanto, na última avaliação a IAC-12 apresentavou redução no NF devido principalmente a intoxicações provocadas pelo herbicida. As demais plantas mantiveram o aumento no NF durante $\mathrm{o}$ período de avaliação, sendo indicativo de que o mesotrione não causou queda de folhas das plantas apesar da intoxicação visível. Essa é uma característica desejada, pois a manutenção da folhagem das plantas de mandioca aumenta o período de atividade do aparato fotossintético da planta proporcionando incrementos no rendimento da produção da cultura. Segundo Sangoi \& Kruse (1993), o aumento de folhas das plantas de mandioca ocorre de forma exponencial até aproximadamente 150 dias após a emergência, sendo que nesse período ocorre grande parte do acúmulo de matéria seca das raízes e parte aérea da cultura.

Tabela 4 - Número de folhas de variedades de mandioca aos 7, 14, 28 e 35 dias após a aplicação (DAA) do mesotrione.

\begin{tabular}{|c|c|c|c|c|c|c|c|c|}
\hline \multirow{3}{*}{ Cultivares } & 7 DAA & 14 DAA & 28 DAA & \multicolumn{5}{|c|}{35 DAA } \\
\hline & \multirow{2}{*}{ Média } & \multirow{2}{*}{ Média } & \multirow{2}{*}{ Média } & \multicolumn{5}{|c|}{ Dose (g i.a. ha $\left.{ }^{-1}\right)$} \\
\hline & & & & $\mathbf{0}$ & 0,072 & 0,108 & 0,144 & 0,216 \\
\hline Cacau UFV & $19,0 \quad \mathrm{C}$ & $25,5 \quad \mathrm{C}$ & $23,5 \quad \mathrm{BC}$ & $31,7 \mathrm{Ab}$ & $20,6 \mathrm{ABc}$ & $40,5 \mathrm{Aa}$ & $19,9 \mathrm{Ac}$ & $25,9 \mathrm{BCbc}$ \\
\hline Coimbra & $24,7 \mathrm{AB}$ & $32,4 \mathrm{AB}$ & $30,1 \mathrm{AB}$ & $31,9 \mathrm{Aa}$ & $24,6 \mathrm{ABa}$ & $30,4 \mathrm{Ba}$ & $24,3 \mathrm{Aa}$ & $26,6 \mathrm{ABa}$ \\
\hline Coqueiro & $21,6 \mathrm{BC}$ & $28,6 \quad \mathrm{BC}$ & $25,0 \quad \mathrm{BC}$ & $35,5 \mathrm{Aa}$ & $26,4 \mathrm{Ab}$ & $29,1 \mathrm{Bab}$ & $25,9 \mathrm{Ab}$ & $22,1 \quad \mathrm{BCb}$ \\
\hline IAC-12 & $28,0 \mathrm{~A}$ & $37,6 \mathrm{~A}$ & $34,1 \mathrm{~A}$ & $27,3 \mathrm{Aa}$ & $16,3 \mathrm{Bb}$ & $25,9 \mathrm{Ba}$ & 18,8 Aab & 16,9 \\
\hline Platina & $20,0 \quad \mathrm{C}$ & $27,6 \quad \mathrm{BC}$ & 22,3 & $35,1 \mathrm{Aab}$ & $26,6 \mathrm{Abc}$ & $42,2 \mathrm{Aa}$ & $21,8 \mathrm{Ac}$ & $35,0 \mathrm{Aab}$ \\
\hline CV (\%) & 21,42 & 21,38 & 28,13 & & ----------- & 16,02 & - & --- \\
\hline
\end{tabular}

Para as variáveis, matéria seca de folhas (MSF), caule (MSC), raízes (MSR) e total (MST) constatou-se que somente o fator cultivares atuou de forma significativa (Tabela 5). Assim os efeitos observados nos cultivares devem ser atribuídos às diferenças genotípicas destas e não à ação dos tratamentos químicos. Esses resultados mostram que apesar de causar intoxicações visíveis às plantas, o mesotrione não causou danos no acúmulo de biomassa dos componentes vegetativos da mandioca, indicativo de alta tolerância da cultura a esse herbicida. O cultivar Coqueiro apresentou os maiores resultados quando comparado às demais para MSF, MSC, MSR e MST (Tabela 5). De modo contrário, o cultivar IAC-12 obteve os menores valores nas variáveis analisadas. Deve-se ressaltar que as manivas dessa cultivar apresentavam os menores diâmetros (dados não apresentados), o que possivelmente afetou os valores de MSR e consequentemente a MST.

Para o cultivar coqueiro foi observado os maiores valores de área foliar (AF) e os menores para a Coimbra (Tabela 5). Apesar de causar o branqueamento às folhas da mandioca o mesotrione não promoveu reduções significativas dessa variável em nenhuma das cultivares. A manutenção da AF pelas plantas de mandioca é uma característica desejada, pois segundo Cruz \& Pelacani (1993) a queda dessa variável e, consequentemente, a redução no índice de área foliar estão diretamente relacionados a reduções no acúmulo de matéria seca pelas raízes e parte área da 
Silveira et al.

cultura e atraso na formação e diminuição no crescimento da raiz tuberosa.

Tabela 5 - Valores médios de matéria seca de folha (MSF), caule (MSC), raízes (MSR), total (MST) e área foliar (AF) de cultivares de mandioca aos 35 dias após a aplicação (DAA) do mesotrione.

\begin{tabular}{|c|c|c|c|c|c|}
\hline \multirow{3}{*}{ Cultivares } & \multicolumn{5}{|c|}{ Variáveis } \\
\hline & MSF & MSC & MSR & MST & $\mathbf{A F}$ \\
\hline & \multicolumn{4}{|c|}{ g/planta } & $\mathrm{cm}^{2}$ \\
\hline Cacau UFV & $12,3 \quad \mathrm{~B}$ & $14,1 \quad \mathrm{~B}$ & $28,3 \mathrm{~B}$ & $54,8 \quad$ B & $4291,0 \mathrm{AB}$ \\
\hline Coimbra & $10,7 \quad C D$ & $15,5 \mathrm{AB}$ & $35,7 \mathrm{~A}$ & $61,9 \mathrm{AB}$ & 3775,1 \\
\hline Coqueiro & $13,7 \mathrm{~A}$ & $17,1 \mathrm{~A}$ & $35,8 \mathrm{~A}$ & 66,6 A & $4749,0 \mathrm{~A}$ \\
\hline IAC-12 & 9,8 & $15,0 \mathrm{AB}$ & $17,1 \quad \mathrm{C}$ & $41,8 \quad \mathrm{C}$ & $3967,5 \quad \mathrm{BC}$ \\
\hline Platina & $11,6 \quad \mathrm{BC}$ & $14,9 \mathrm{AB}$ & 27,3 B & $53,8 \quad$ B & $4060,0 \quad \mathrm{BC}$ \\
\hline CV (\%) & 10,22 & 18,25 & 27,12 & 17,24 & 13,49 \\
\hline
\end{tabular}

- Médias seguidas por mesmas letras maiúsculas na coluna não diferem entre si pelo teste de Tukey $(\mathrm{p} \leq 0,05)$. CV: Coeficiente de Variação

\section{Conclusões}

Os cultivares de mandioca apresentaram elevada tolerância ao herbicida mesotrione, não sendo relatadas reduções no crescimento inicial da cultura. No entanto, são necessários novos estudos em nível de campo para determinar época e modo de aplicação do mesotrione e seus efeitos sobre a produtividade final da cultura.

\section{Agradecimentos}

A Coordenação de Aperfeiçoamento de Pessoal de Nível Superior (CAPES), Conselho Nacional de Desenvolvimento Científico e Tecnológico $(\mathrm{CNPq})$ e a Fundação de Amparo à Pesquisa do Estado de Minas Gerais (FAPEMIG) pelo apoio financeiro na execução deste trabalho.

\section{Referências}

ALBUQUERQUE, J.A.A. et al. Interferência de plantas daninhas sobre a produtividade da mandioca (Manihot esculenta). Planta Daninha, v.26, n.2, p.279-289, 2008.

BIFFE, D.F. et al. Avaliação de herbicidas para dois cultivares de mandioca. Planta Daninha, v.28, n.4, p.807-816, 2010.
CARDOSO, C.E.L.; SOUZA, J.S. Importância, potencialidades e perspectivas do cultivo da mandioca na América Latina. In: CEREDA, M.P. Culturas de tuberosas amiláceas latino americanas. Campinas: Fundação Cargill, 2002. p.29- 47.

CARVAlHO, J.E.B. et al. Período de controle de plantas infestantes na cultura da mandioca no Estado da Bahia. Cruz das Almas: Embrapa, 2004. 7p. (Comunicado Técnico, 109).

CRUZ, J.L.; PELACANI, R. Fisiologia da mandioca. In: Curso nacional de mandioca, 8., 1993, Cruz das Almas. EMBRAPACNPMF, 1993. 38 p.

GUGLIERI, A. et al. Fitossociologia de plantas espontâneas em um mandiocal implantado em pastagem cultivada em Mato Grosso do Sul, Brasil. Revista de Ciências Agrárias, v.51, n.1, p.127-141, 2009.

JOHANNS, O.; CONTIERO, R. Efeitos de diferentes períodos de controle e convivência de plantas daninhas com a cultura da mandioca. Revista Ciência Agronômica, v.37, n.3, p.326-331, 2006.

MAPA.http://extranet.agricultura.gov.br/agrofi t_cons/principal_agrofit_cons. Ministério da 
Silveira et al.

Agricultura, Pecuária e Abastecimento Coordenação-Geral de Agrotóxicos e Afins. Acesso em: 01/08/2011.

MOURA, G.M. Interferência de plantas daninhas na cultura da mandioca (Manihot esculenta Crantz) no Estado do Acre. Planta Daninha, v.18, n.3, p.451-456, 2000.

OLIVEIRA Jr., R.S. et al. Tolerância de cinco cultivares de mandioca (Manihot esculenta) a herbicidas. Planta Daninha, v.19, n.1, p.119125, 2001.

PINOTTI, E.B. et al. Levantamento florístico de plantas daninhas na cultura da mandioca no município de Pompéia - SP. Revista Raízes e Amidos Tropicais, v.6, n.1, p.120-125, 2010. RODRIGUES, B.N.; ALMEIDA, F.R. Guia de herbicidas. 5.ed. Londrina: Edição dos Autores, 2005. 591 p.

SANGOI, L.; KRUSE, N.D. Acúmulo e distribuição de matéria seca em diferentes frações da planta de mandioca no planalto catarinense. Pesquisa Agropecuária Brasileira, v.28, n.10, p.1151-1164, 1993.

VIDIGAL FILHO, P.S. et al . Avaliação de cultivares de mandioca na Região Noroeste do Paraná. Bragantia, v.59, n.1, p.69-75, 2000. 